\title{
Specialty choice among dental students in Ibadan, Nigeria
}

\author{
K K Kanmodi, ${ }^{1}$ Dental student; A I Badru, ${ }^{2}$ MBBS, FMCA; A G Akinloye, ${ }^{3}$ BEd, MEd; W A Wegscheider, ${ }^{4}$ MD \\ ${ }^{1}$ Faculty of Dentistry, University of Ibadan, Ibadan, Nigeria \\ ${ }^{2}$ Department of Anaesthesia, Faculty of Clinical Sciences, Olabisi Onabanjo University, Sagamu, Nigeria \\ ${ }^{3}$ Department of Mathematics and Statistics, Faculty of Science, Osun State College of Technology, Esa Oke, Nigeria \\ ${ }^{4}$ Department of Dentistry and Maxillofacial Surgery, Medical University of Graz, Graz, Austria
}

Corresponding author: K K Kanmodi (kanmodikehinde@yahoo.com)

\begin{abstract}
Background. The unequal distribution of workforce across dental specialties in Nigeria poses a significant problem in the delivery of specialists' oral healthcare to the Nigerian population.

Objectives. To determine dental specialties preferences among dental students at the University of Ibadan, Nigeria, and to explore the factors that influence their choices.

Methods. We obtained ethical approval to conduct this study. Only the dental students who rotated through all the dental specialties were selected to participate in this questionnaire-based study. Data were analysed using SPSS version 16 (SPSS Inc., USA).

Results. The majority of dental students at the University of Ibadan preferred the oral and maxillofacial surgery (OMS) specialty above all other dental specialties, while prosthetic dentistry was least preferred. Of all the factors to take into consideration when choosing a dental specialty, personal interest was the only factor considered by nearly all respondents. Only male respondents considered prestige as an influencing factor in their choice of a specialty. Lifestyle and job description were factors considered by a higher proportion of the male respondents (10/13) than females (5/14). The mean age of the 27 respondents who participated in this study was 22.6 years, $52 \%$ of whom were females.

Conclusion. OMS was the most preferred specialty among our respondents $(n=8)$. Nearly all dental students chose residency training in the specialty that most appealed to them. The interest of dental students towards the least appealing dental specialties needs to be developed to solve the problem of skewed distribution of the dental workforce in Nigeria. Our findings suggest that this may be accomplished by changing dental students' perceptions of certain specialties, building on male students' interests in job security and private practice potential, and the female students' interests in family-friendly specialties and increasing flexibility in dental residency programmes.
\end{abstract}

Afr J Health Professions Educ 2017;9(1):21-23. DOI:10.7196/AJHPE.2017.v9i1.670

There is a persistent shortage and an unequal distribution of workforce across the dental specialties in Nigeria. ${ }^{[1-3]}$ This has been attributed to biased levels of interest by dental graduates towards all dental specialties, as a few specialties are preferred above others. ${ }^{[1]}$ Oral and maxillofacial surgery (OMS) has been reported as the most preferred specialty among the majority of Nigerian dental graduates, while prosthetic dentistry (PROS) is the least preferred. ${ }^{[1]}$ This is a significant problem, as some dental specialties persistently have greater manpower than other dental specialties, which hinders the adequate delivery of holistic oral healthcare to the Nigerian population. ${ }^{[1-3]}$

Dental students' and graduates' choice of a dental specialty is determined by many factors, including potential financial income, self-employability, personal interest, job security, fear of litigation, and prestige. . $^{[4,5]}$

The trend of specialty choice among dental students at the University of Ibadan, Nigeria has yet to be determined. The objective of this study was to determine the most preferred dental specialties among these dental students, and also to explore the factors that inform their choice. Our findings try to provide solutions to the problem of unequal preference of dental specialties.

\section{Methods}

Ethical approval to carry out this study was obtained from the Oyo State Ministry of Education, Nigeria. The study population comprised dental students in the Faculty of Dentistry, University of Ibadan. Only students who rotated through all the academic departments within the faculty were considered eligible to participate in the study. Based on this criterion, only students in their final year were able to participate.

The study tool was a 34-item questionnaire that was self-administered to the participants after obtaining verbal informed consent. Twenty-seven final-year dental students, of a total of 35 eligible students, volunteered to participate in the study. The questionnaire had three sections:

- Section A obtained information on the sociodemographic data of the participants.

- Section B obtained information on the choice of dental specialties being considered by participants for a residency programme.

- Section C obtained information on the factors that informed participants' choice of a dental specialty.

Collected data were statistically analysed using SPSS version 16.0 (SPSS Inc., USA). The frequencies, proportions, arithmetic means, and standard deviations of variables were determined and are illustrated using a table and a chart. Tests of association between qualitative variables were done using the $\chi^{2}$ test; $p<0.05$ was considered statistically significant.

\section{Results}

The response rate was $77.1 \%$ (27/35). The mean age of the respondents was 22.6 years, and the gender distribution was fairly even, with 13 males (48\%) and 14 females (52\%) participating. 


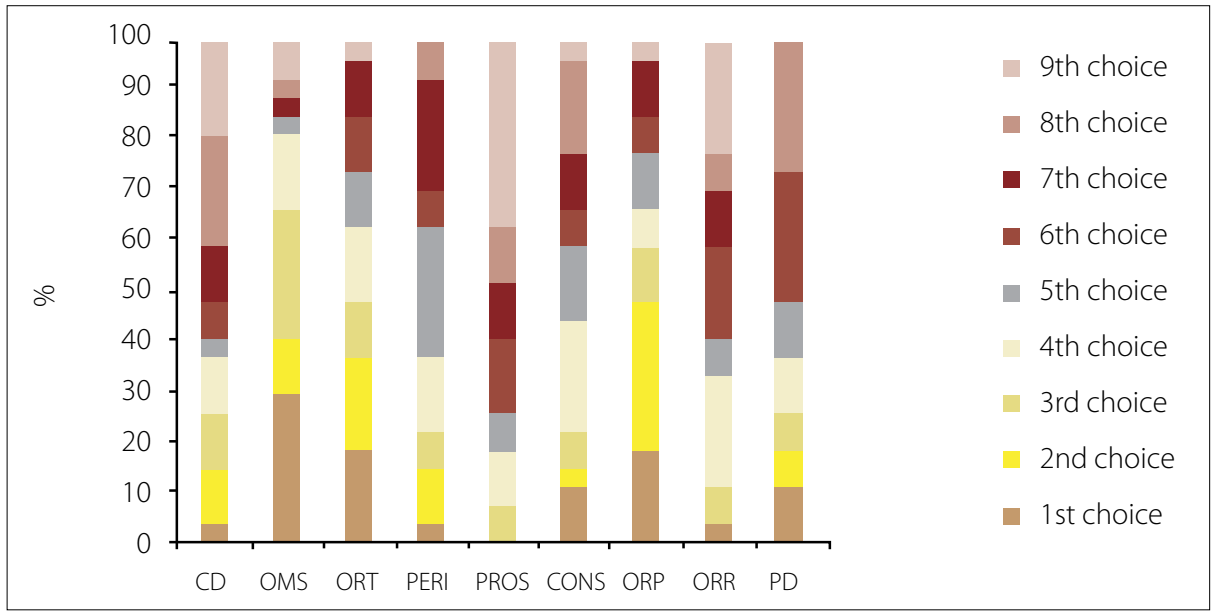

Fig. 1. The percentage distribution of specialty choices (in ranking) among respondents. (CD = community dentistry; OMS = oral and maxillofacial surgery; ORT = orthodontics; PERI = periodontology; PROS = prosthetic dentistry; CONS = conservative dentistry; ORP = oral pathology; ORR = oral radiology; $P D=$ paediatric dentistry.)

OMS was the specialty with the highest frequency of being the first choice $(8 / 27(29.6 \%))$ of the respondents, all of whom were considering a residency training programme after graduation. Oral pathology (ORP), OMS, and orthodontics (ORT) were the top three 1st and 2nd choice specialties. The least popular specialty choices, placed 7th, 8th, and 9th, were PROS, commu- nity dentistry (CD), and oral radiology (ORR) (Fig. 1).

Table 1 shows the comparison between gender and factors influencing the choice of our respondents for any dental specialty. More than half of the male respondents chose a specialty based on the prestige associated with it, while none of the female respondents made a choice based on that factor $(7 / 13$ v. $0 / 14, p=0.001)$. A higher proportion of the male respondents compared with the female respondents considered lifestyle (including job description) as a factor (10/13 v. 5/14, $p=0.031$ ). Further comparisons of association between gender of respondents and other influencing factors were not statistically significant (Table 1).

For men, the top influential factors were personal interest (100\%), lifestyle/job description (76.9\%), and job security/potential for advancement and opportunity for private practice (61.5\% each).

For women, the top influential factor, as with men, was personal interest $(85.7 \%)$, which was the only factor chosen by more than half of the women. The next highest influencing factors for women were easy compatibility with having a family and enough time for leisure activity (42.9\% each).

All the males and females indicated that ease of entry into the residency programme and gender distribution within the specialty had no influence on their decision-making. Prestige, others' perception of the job, and low risk of litigation were also chosen by all female participants $(100 \%)$ as having no influence on their choices.

Table 1. Comparison between gender distribution and factors influencing specialty choice among respondents

\begin{tabular}{|c|c|c|c|c|c|}
\hline \multirow[b]{2}{*}{ Influencing factors } & \multicolumn{2}{|c|}{ Male $(n=13)$} & \multicolumn{2}{|c|}{ Female $(n=14)$} & \multirow[b]{2}{*}{$\chi^{2}(p$-value $)$} \\
\hline & Yes, $n(\%)$ & No, $n(\%)$ & Yes, $n(\%)$ & No, $n(\%)$ & \\
\hline Personal interest in the specialty & $13(100)$ & $0(0)$ & $12(85.7)$ & $2(14.3)$ & 0.157 \\
\hline Job security/advancement prospects & $8(61.5)$ & $5(38.5)$ & $5(35.7)$ & $9(64.3)$ & 0.180 \\
\hline Ease of entry into the residency programme of the specialty & $0(0)$ & $13(100)$ & $0(0)$ & $14(100)$ & * \\
\hline Lifestyle and job description & $10(76.9)$ & $3(23.1)$ & $5(35.7)$ & $9(64.3)$ & 0.031 \\
\hline Prestige & $7(53.8)$ & $6(46.2)$ & $0(0)$ & $14(100)$ & 0.001 \\
\hline Close relation with other specialties & $5(38.5)$ & $8(61.5)$ & $2(14.3)$ & $12(85.7)$ & 0.152 \\
\hline Positive influence during the posting in the specialty & $7(53.8)$ & $6(46.2)$ & $5(35.7)$ & $9(64.3)$ & 0.343 \\
\hline Opportunity to conduct research & $5(38.5)$ & $8(61.5)$ & $2(14.3)$ & $12(85.7)$ & 0.152 \\
\hline Flexibility with training & $7(53.8)$ & $6(46.2)$ & $4(28.6)$ & $10(71.4)$ & 0.182 \\
\hline Amount of patient contact & $5(38.5)$ & $8(61.5)$ & $1(7.1)$ & $13(92.9)$ & 0.050 \\
\hline Technically challenging specialty & $2(15.4)$ & $11(84.6)$ & $4(28.6)$ & $10(71.4)$ & 0.410 \\
\hline High wages & $6(46.2)$ & $7(53.8)$ & $4(28.6)$ & $10(71.4)$ & 0.345 \\
\hline Opportunity for private practice & $8(61.5)$ & $5(38.5)$ & $4(28.6)$ & $10(71.4)$ & 0.085 \\
\hline Easy compatibility with having a family & $4(30.8)$ & $9(69.2)$ & $6(42.9)$ & $8(57.1)$ & 0.516 \\
\hline Inclination of specialty before entering dental school & $1(7.7)$ & $12(92.3)$ & $4(28.6)$ & $10(71.4)$ & 0.163 \\
\hline A wide variety of caseload & $3(23.1)$ & $10(76.9)$ & $2(14.3)$ & $12(85.7)$ & 0.557 \\
\hline Influence from a mentor & $6(46.2)$ & $7(53.8)$ & $3(21.4)$ & $11(78.6)$ & 0.173 \\
\hline Enough time left for leisure activity & $4(30.8)$ & $9(69.2)$ & $6(42.9)$ & $8(57.1)$ & 0.516 \\
\hline Influence of family or relative & $1(7.7)$ & $12(92.3)$ & $1(7.1)$ & $13(92.9)$ & 0.957 \\
\hline Other people's perception of the job & $1(7.7)$ & $12(92.3)$ & $0(0)$ & $14(100)$ & 0.290 \\
\hline Little on-call commitment & $2(15.4)$ & $11(84.6)$ & $1(7.1)$ & $13(92.9)$ & 0.496 \\
\hline Low risk of litigation & $3(23.1)$ & $10(76.9)$ & $0(0)$ & $14(100)$ & 0.057 \\
\hline Gender distribution in the specialty & $0(0)$ & $13(100)$ & $0(0)$ & $14(100)$ & * \\
\hline
\end{tabular}




\section{Discussion}

OMS is known to be one of the most preferred specialties among dental students and graduates. ${ }^{[1,4]}$ This specialty was also the most appealing specialty among our study population. Our respondents showed little interest in specialising in PROS, CD, and ORR. This indicates a high possibility that the number of prosthetic dental specialists, community dentists and oral radiologists will remain lower than all other dental specialists in the country.

There were different factors that informed our respondents' choice of a dental specialty (Table 1). We noticed that personal interest played an important role, as almost all of our respondents selected it as a major factor in their selection of a specialty. Personal interest had also been reported as a major influential factor with regard to the choice of a medical specialty among some final-year medical students at the University of Calabar, Nigeria. ${ }^{[6]}$ This reveals that most students prefer the residency training programmes that are most appealing to them, but it would be helpful to know how these personal interests are formed and how they could be influenced.

Prestige, lifestyle/job description, and low risk of litigation were more commonly considered among males than females. Addressing potential misconceptions about each dental speciality during student rotations may contribute to a broader range of acceptable choices for male dental students and may change personal interests for both genders. Job security and potential for private practice were also important factors for male students. Both of these factors are strongest in specialties with fewer competitors. Pointing out that the less desirable specialties may have the highest job security and private practice potential might influence male students to consider specialities they might have otherwise overlooked. Combining a residency training programme with family life was a more common concern among female dental trainees. The creation of residency programmes with a more flexible family-friendly structure may encourage female dental graduates to go into specialties where females tend to be under-represented or where there are shortages.

Furthermore, the gender distribution of existing specialists in a dental specialty, call duties, technicalities associated with specialist training, perception of people about a specialty, and ease of admission into the residency programme of a specialty, among others (Table 1), did not seem to have much influence on dental students' choice of a specialty. Changes made to these factors would have less impact in attracting male or female residents to specialties.

\section{Conclusion}

We found that the majority of Ibadan dental students want to specialise in OMS, ORT, or ORP because these specialties appeal most to their personal interests. Many dental students were least interested in becoming a prosthetic dental specialist, community dentist or oral radiologist. We conclude that there is still a strong likelihood that PROS and other less appealing specialties may remain under-staffed in the future. To prevent this, dental students' interest must be stimulated by changing their perceptions of certain specialties, building on their interests in job security and potential for private practice and, particularly for female dentists, by increasing flexibility in dental residency programmes.

Acknowledgement. The authors appreciate the assistance of Dr K E Shields, Shields Medical Writing, Greater Philadelphia area, USA, for her in-depth review of this research and manuscript. The principal investigator (KKK) also thanks Miss O M Familoni and Mr M A Owadokun for their inspiration and support.

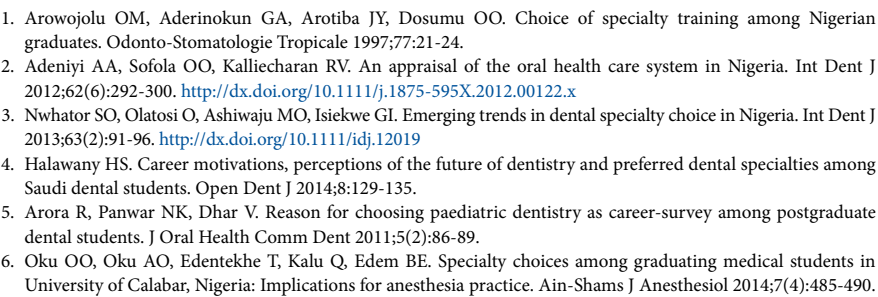

6. Oku OO, Oku AO, Edentekhe T, Kalu Q, Edem BE. Specialty choices among graduating medical students in University of Calabar, Nigeria: Implications for anesthesia practice. Ain-Shams I Anesthesiol 2014;7(4):485-490. http://dx.doi.org/10.4103/1687-7934.145673 